International Journal of Pure and Applied Mathematics

Volume 108 No. 1 2016, 63-79

ISSN: 1311-8080 (printed version); ISSN: 1314-3395 (on-line version)

url: http://www.ijpam.eu

doi: 10.12732/ijpam.v108i1.8

\title{
HAAR WAVELETS BASED TIME DISCRETIZATION TECHNIQUE FOR SOLVING NONLINEAR PARTIAL DIFFERENTIAL EQUATIONS
}

\author{
Harpreet Kaur ${ }^{1}$, Shin Min Kang ${ }^{2} \S$ \\ ${ }^{1}$ Department of Mathematics \\ School of Physical Sciences \\ Lovely Professional University \\ Phagwara, 144411, Punjab, INDIA \\ ${ }^{2}$ Department of Mathematics and RINS \\ Gyeongsang National University \\ Jinju 52828, KOREA
}

\begin{abstract}
A new numerical technique is developed to find the solutions of general nonlinear partial differential equations. The technique is based on the time discretization of Haar wavelet series approximations with quasilinearization process. In order to test the efficiency of the proposed technique, it is applied on well known nonlinear partial differential equations such as the generalized regularized long wave equation, the Benjamin Bona-Mahony equation and the Fitzhugh-Nagumo equation. Numerical results are obtained by preparing MATLAB codes of proposed techniques. The beautiful concentration profiles of $u$ and $v$ are shown by figures at different time level and error norms $L_{2}$ and $L_{\infty}$ are calculated.
\end{abstract}

AMS Subject Classification: 35Qxx, 41A65, 65Nxx, 65T60

Key Words: Haar wavelet, operational matrix, nonlinear partial differential equation, quasilinearization process, time discretization of Haar wavelet series

\section{Introduction}

Partial differential equations form the basis of many mathematical models of

Received: March 9, 2016

Published: May 31, 2016

$\S_{\text {Correspondence author }}$ (c) 2016 Academic Publications, Ltd.

url: www.acadpubl.eu 
physical, chemical and biological phenomena and more recently their use has spread into economics, financial forecasting, image processing and other fields. The vast majority of partial differential equation models cannot be solved analytically. So, to investigate the predictions of partial differential equation models of such phenomena it is often necessary to approximate their solution numerically. In most cases, the approximate solution is represented by functional values at certain discrete points (grid points or mesh points). Currently, there are many numerical techniques available in the literature. Among them, the finite difference, finite element, and finite volume methods fall under the category of low order methods, whereas spectral and pseudo spectral methods are considered global methods. Sometimes the latter two methods are considered as subsets of the method of weighted residuals. In this paper numerical solutions of the nonlinear partial differential equations like as the damped generalized regularized long wave equation, the Benjamin-Bona-Mahony-Burgers equation and the Fitzhugh-Nagumo equation in one space dimension are considered. The mathematical model of propagations of small-amplitude long waves in nonlinear dispersive media is described by the Benjamin-Bona-Mahony-Burgers equation [19] and generalized regularized long wave equation [10]. For mathematical and physical significance of these equations, one may be able to refer [7, 17]. Mei [15] has been discussed large times behavior of solutions of the BenjaminBona-Mahony-Burgers equation. Yousefi et al. [22] have found the solutions of the damped generalized regularized long wave equation by Bernstein RitzGalerkin. The Fitzhugh-Nagumo equation has various applications in the fields of flame propagation, logistic population growth, neurophysiology, branching Brownian motion process, autocatalytic chemical reaction and nuclear reactor theory (see [5, 18]). Recently, Triki and Wazwaz [21] considered the generalized Fitzhugh-Nagumo equation exhibiting time-varying coefficients and linear dispersion terms.

Now we present a new Haar wavelet based time discretization schemes to find the solution of well known nonlinear partial differential equations. The method is based upon Haar wavelet approximation and quasilinearization process.

The paper is organized as follows: In Section 2, we introduce a given Haar wavelet function and Section 3 describes the basic formulation of numerical schemes for our subsequent development. Section 4 is devoted to the solution of nonlinear partial differential equations by using Haar wavelet approximations and we report our numerical findings and demonstrate the accuracy of the proposed scheme. Section 5 consists brief conclusion of presented work. 


\section{Haar Wavelet Functions}

We shall begin our introduction of wavelets with multiresolution analysis. This provides the mathematical foundation of the construction of wavelets and wavelet bases, and naturally leads to the description of the scaling and wavelet function. The idea of multiresolution analysis is similar to sub-band decomposition and coding, which divides a signal into a set of frequency bands in a particular way for efficiency [20]. Multiresolution analysis provides the means of looking at the fine detail of a signal, or one can obtain an overall sense of the behavior. From multiresolution analysis one can then develop the filters associated with wavelets that leads naturally to the discrete wavelet transform, so we may construct an orthonormal basis for $L^{2}(R)$. Multiresolution analysis provides a powerful framework for analyzing functions at various levels of detail or resolution.

Now we introduce Haar wavelet reconstructions and decompositions.

Multiresolution analysis entails a sequence of nested closed approximation subspaces $V_{j}=\left\{f \in L^{2}(R): f\right.$ is a constant on $\left.\left[2^{j} k, 2^{j}(k+1)\right], k \in Z\right\}$, the family of Haar wavelets utilizes the concept of multiresolution analysis. The increasing sequence $\left\{V_{j}\right\}_{j \in Z}$ of subsets of $L^{2}(R)$ with the scaling function $\phi$ is called multiresolution analysis.

The coefficients are calculated as

$$
\begin{aligned}
a_{j, k} & =\left\langle f, \phi_{j, k}\right\rangle=2^{-j} \int_{2^{\mathrm{j}} k}^{2^{\mathrm{j}}(k+1)} f(x) d x \\
a_{j+1, k} & =\frac{1}{\sqrt{2}}\left(a_{j, 2 k}+a_{j, k+1}\right) \quad \text { since } V_{j+1}=V_{j} \oplus W_{j} .
\end{aligned}
$$

The projection $P_{j} f$ defined as

$$
\begin{aligned}
\left(P_{j}-P_{j-1}\right) f & =\frac{1}{\sqrt{2}} \sum\left(a_{j, 2 k}-a_{j, 2 k+1}\right)\left(\phi_{j, 2 k}-\phi_{j, 2 k+1}\right), \\
P_{j} f & =\sum a_{j k} \phi_{j k} .
\end{aligned}
$$

Also functions

$$
\begin{aligned}
\Psi(t) & =\phi_{2 t}-\phi_{2 t-1}= \begin{cases}1 & 0 \leq t<\frac{1}{2}, \\
-1 & \frac{1}{2} \leq t<1, \\
0 & \text { otherwise },\end{cases} \\
\Psi_{j, k+1} & =\frac{1}{\sqrt{2}}\left(\phi_{j, 2 k}-\phi_{j, 2 k+1}\right), \\
Q_{j+1} f & =\left(P_{j}-P_{j+1}\right) f=\sum d_{j+1, k} \phi_{j+1, k},
\end{aligned}
$$


where

$$
d_{j+1, k}=\left\langle f, \Psi_{j+1, k}\right\rangle=\frac{1}{\sqrt{2}}\left(a_{j, 2 k}-a_{j, 2 k+1}\right) .
$$

The basic and simplest form of Haar wavelets is the Haar scaling function that appears in the form of a square wave over the interval $[0,1]$, generally written as

$$
h_{1}(x)= \begin{cases}1 & x \in[0,1) \\ 0 & \text { elsewhere }\end{cases}
$$

The above expression, called Haar father wavelets, is the zero ${ }^{\text {th }}$ level wavelet which has no displacements and dilations of unit magnitude. Wavelets can produce coefficients where many are zero and only a few are non-zeros. Wavelets are able to examine functions locally. The following definitions illustrate the translation dilation of wavelet functions. Thus we can write out the Haar wavelet family as

$$
h_{i}(x)=h_{i}\left(2^{j} x-k\right)= \begin{cases}1, & \frac{k}{2^{\jmath}} \leq x<\frac{k+0.5}{2^{\jmath}} \\ -1, & \frac{k+0.5}{2^{\jmath}} \leq x<\frac{k+1}{2^{\jmath}} \\ 0 & \text { elsewhere }\end{cases}
$$

for $i \geq 2, i=2^{j}+k+1, j \geq 0,0 \leq k \leq 2^{j}-1$ and collocation points $x_{l}=\frac{l-0.5}{2 m}, l=1,2 \ldots 2 m$. Here $m$ is the level of the wavelet, we assume the maximum level of resolutions is index $J$. Thus $m=2^{j}(j=0,1,2, \ldots, J)$; in case of minimal values $m=1, k=0$, then $i=2$. For any fixed level $m$, there are $m$ series of $i$ to fill the interval $[0,1)$ corresponding to that level and for a provided $J$, the index number $i$ can reach the maximum value $M=2^{J=1}$, when including all levels of wavelets. The operational matrices $p_{i, 1}(t)$ and $p_{i, 2}(t)$ of order $2 m \times 2 m$ can be obtained by integrations of Haar wavelets in the following forms (see $[2,11])$.

$$
p_{i, 1}(x)= \begin{cases}t-\frac{k}{m}, & \frac{k}{m} \leq x<\frac{k+0.5}{m} \\ \frac{k+1}{m}-t, & \frac{k+0.5}{m} \leq x<\frac{k+1}{m} \\ 0 & \text { elsewhere }\end{cases}
$$

and

$$
p_{i, 2}(x)= \begin{cases}\frac{1}{2}\left(x-\frac{k}{m}\right)^{2}, & \frac{k}{m} \leq x<\frac{k+0.5}{m} \\ \frac{1}{4 m^{2}}-\frac{1}{2}\left(\frac{k+1}{m}-x\right)^{2}, & \frac{k+0.5}{m} \leq x<\frac{k+1}{m} \\ \frac{1}{4 m^{2}}, & \frac{k+1}{m} \leq x<1 \\ 0 & \text { elsewhere }\end{cases}
$$


The quasilinearization process [13] is an application of the Newton-RaphsonKantrovich approximation method in a function space. The process is applied to solve a nonlinear $n^{\text {th }}$ order ordinary or partial differential equation in $N$ dimensions as a limit of a sequence of linear differential equations. The quasilinearization prescription determines the iterative approximation to the solution of a given equation and linearized the equation. The zero ${ }^{t h}$ approximation $u_{0}(x, t)$ is chosen from mathematical or physical considerations. Kaur et. al have proposed Haar wavelet quasilinearization approach for solving nonlinear boundary value problems $[8,9]$ and space discretization scheme for solving coupled Burger equations $[16]$.

\section{Numerical Schemes for Solving the Nonlinear Partial Differential Equations}

The orthogonality property puts a strong limitation on the construction of wavelets and allows us to transform any square integral function on the interval time $[0,1)$ into the Haar wavelets series as

$$
f(x)=a_{0} h_{0}(x)+\sum_{j=0}^{\infty} \sum_{k=0}^{2^{\mathrm{j}}-1} a_{2^{\mathrm{j}}+k} h_{2^{\mathrm{j}}+k}(x), \quad x \in[0,1] .
$$

Similarly the highest derivative can be written as wavelet series $\sum_{i=-\infty}^{\infty} a_{i} h_{i}(x)$. In applications, The Haar series is always truncated to $2 m$-terms and we assume as

$$
\begin{gathered}
\dot{u}^{\prime \prime}\left(x_{l}, t\right)=\sum_{i=0}^{2 m} a_{i}(t) h_{i}\left(x_{l}\right), \\
u^{\prime \prime}(x, t)=\left(t-t_{s}\right) \sum_{i=0}^{2 m} a_{i} h_{i}(x)+u^{\prime \prime}\left(x, t_{s}\right), \\
u(x, t)=\left(t-t_{s}\right) \sum_{i=0}^{2 m} a_{i} p_{2}(x)+u(0, t)+u(x, t s)-u\left(0, t_{s}\right) \\
+x\left(u^{\prime}(0, t)-u^{\prime}\left(0, t_{s}\right)\right), \\
u(x, t)=\sum_{i=0}^{2 m} a_{i} p_{2}(x)+u(0, t)+x u^{\prime}(0, t), \\
\left.u\left(0, t_{s}\right)=f_{1}^{\prime}\left(t_{s}\right), \quad u\left(1, t_{s}\right)\right)=f_{2}\left(t_{s}\right), \\
u(0, t)=f_{1}^{\prime}(t), \quad u(1, t)=f_{2}^{\prime}(t),
\end{gathered}
$$




$$
\dot{u}(x, t)=\sum_{i=0}^{2 m} a_{i} p_{2}(x)+\dot{u}(0, t)+x \dot{u}^{\prime}(0, t) .
$$

By substituting $x=1$ in (3.1c) and (3.1d),

$$
\begin{aligned}
&\left(u^{\prime}(0, t)-u^{\prime}\left(0, t_{s}\right)\right)=-\left(t-t_{s}\right) \sum_{i=0}^{2 m} a_{i} p_{2}(1)+f_{2}(t)-f_{1}(t) \\
&-f_{2}\left(t_{s}\right)+f_{1}\left(t_{s}\right), \\
& \dot{u}^{\prime}(0, t)= f_{2}^{\prime}(t)-\sum_{i=0}^{2 m} a_{i} p_{2}(1)-f_{1}^{\prime}(t), \\
& u^{\prime}(0, t)=f_{2}^{\prime}(t)-\sum_{i=0}^{2 m} a_{i} p_{2}(1)-f_{1}^{\prime}(t) .
\end{aligned}
$$

Eqs.(3.2a)-(3.2c) and boundary conditions into Eqs.(3.1c)-(3.1d) and discretizing the results by $x \rightarrow x_{l}$ and $t \rightarrow t_{s+1}$,

$$
\begin{aligned}
& \dot{u}^{\prime \prime}\left(x_{l}, t_{s+1}\right)=\sum_{i=0}^{2 m} a_{i} h_{i}\left(x_{l}\right), \\
& u^{\prime \prime}\left(x_{l}, t_{s+1}\right)=\triangle t \sum_{i=0}^{2 m} a_{i} h_{i}\left(x_{l}\right)+u^{\prime \prime}\left(x_{l}, t_{s}\right), \\
& u^{\prime}\left(x_{l}, t_{s+1}\right)=\triangle t \sum_{i=0}^{2 m} a_{i} p_{1}\left(x_{l}\right)-\triangle t \sum_{i=0}^{2 m} a_{i} p_{2}(1)+f_{2}\left(t_{s+1}\right) \\
& -f_{1}\left(t_{s+1}\right)-f_{1}\left(t_{s}\right)+f_{2}\left(t_{s}\right)+u^{\prime}\left(x_{l}, t_{s}\right), \\
& +x_{l}\left(-\triangle t \sum_{i=0}^{2 m} a_{i} p_{2}(1)+f_{2}\left(t_{s+1}\right)-f_{1}\left(t_{s+1}\right)-f_{2}\left(t_{s}\right)+f_{1}\left(t_{s}\right)\right), \\
& \dot{u}\left(x_{l}, t_{s+1}\right)=\triangle t \sum_{i=0}^{2 m} a_{i} p_{2}\left(x_{l}\right)+f_{1}^{\prime}\left(t_{s+1}\right) \\
& +x_{l}\left(-\sum_{i=0}^{2 m} a_{i} p_{2}(1)+f_{2}^{\prime}\left(t_{s+1}\right)-f_{1}^{\prime}\left(t_{s+1}\right)\right) \text {, }
\end{aligned}
$$


where $\triangle(t)=t-t_{s}$.

\section{Numerical Results and Discussions}

In this section, to check the applicability of proposed scheme three examples are considered and the errors $L^{2}$ and $L^{\infty}$ are computed of obtained numerical solutions with following formulas:

$$
L_{2}=\frac{\sqrt{\sum_{l=1}^{2 m}\left|u_{\text {exact }}\left(x_{i}\right)-u_{\text {num }}\left(x_{l}\right)\right|^{2}}}{\sqrt{\sum_{l=1}^{2 m}\left|u_{\text {exact }}\left(x_{i}\right)\right|^{2}}}, \quad L_{\infty}=\max _{l}\left|u_{\text {exact }}\left(x_{i}\right)-u_{\text {num }}\left(x_{l}\right)\right| .
$$

Example 4.1. (The Benjamin-Bona-Mahony-Burger Equation)

The damped generalized regularized long wave equation is a partial differential equation that describes the amplitude of the long wave and written as

$$
u_{t}-\left(\phi(x, t) u_{x t}\right)_{x}-\alpha u_{x x}+\beta u_{x}+u^{p} u_{x}=f(x, t), \quad 0<x \leq 1
$$

with the conditions

$$
u(x, 0)=u_{0}(x), \quad u\left(x_{L}, t\right)=u_{0}\left(x_{R}, t\right)=0, \quad t \in[0, T] .
$$

Consider parameters $\alpha=\beta=p=1$ and $\phi(x, t)=1$ in Eq.(4.1) and got the mathematical model of propagations of small-amplitude long waves in nonlinear dispersive media is described by the Benjamin-Bona-Mahony-Burgers equation $[3,14]$.

$$
u_{t}-u_{x x t}-u_{x x}+u_{x}+u u_{x}=f(x, t) .
$$

Consider Eq.(4.1a) with initial and boundary conditions [1]:

$$
u(x, 0)=\sin x \quad \text { and } \quad u(0, t)=0, \quad u(1, t)=e^{-t} \sin 1
$$

and

$$
f(x, t)=e^{-t}\left(\cos x-\sin x+\frac{1}{2} e^{-t} \sin 2 x\right) .
$$

By using quasilinearization process, Eq.(4.1a) leads to

$$
\begin{aligned}
& \dot{u}\left(x, t_{s+1}\right)-\ddot{u}\left(x, t_{s+1}\right)-u^{\prime \prime}\left(x, t_{s+1}\right)+u^{\prime}\left(x, t_{s+1}\right) \\
& \quad+u^{\prime}\left(x, t_{s}\right) u\left(x, t_{s}\right)+\left(u\left(x, t_{s+1}\right)-u\left(x, t_{s}\right)\right) u^{\prime}\left(x, t_{s}\right) u\left(x, t_{s}\right) \\
& \quad+\left(u^{\prime}\left(x, t_{s+1}\right)-u^{\prime}\left(x, t_{s}\right)\right) u\left(x, t_{s}\right) \\
& =e^{-t_{s}}\left(\cos x-\sin x+\frac{1}{2} e^{-t} \sin 2 x\right) .
\end{aligned}
$$


Using Eqs.(3.1a)-(3.2e) in Eq.(4.1c)

$$
\begin{aligned}
& \triangle t \sum_{i=0}^{2 m} a_{i} p_{i, 2}\left(x_{l}\right)+f_{1}^{\prime}\left(t_{s+1}\right)+x_{l}\left(-\sum_{i=0}^{2 m} a_{i} p_{i, 2}(1)+f_{2}^{\prime}\left(t_{s+1}\right)-f_{1}^{\prime}\left(t_{s+1}\right)\right) \\
& -\sum_{i=0}^{2 m} a_{i} h_{i}\left(x_{l}\right)-\triangle t \sum_{i=0}^{2 m} a_{i} h_{i}\left(x_{l}\right)-u^{\prime \prime}\left(x_{l}, t_{s}\right)+\triangle t \sum_{i=0}^{2 m} a_{i} p_{i, 1}\left(x_{l}\right) \\
& -\triangle t \sum_{i=0}^{2 m} a_{i} p_{i, 2}(1)+f_{2}\left(t_{s+1}\right)-f_{1}\left(t_{s+1}\right)+f_{2}\left(t_{s}\right)-f_{1}(t s)+u^{\prime}\left(x_{l}, t s\right) \\
& +u^{\prime}\left(x_{l}, t_{s}\right)\left(\triangle t \sum_{i=0}^{2 m} a_{i} p_{i, 2}\left(x_{l}\right)+u\left(x_{l}, t_{s}\right)-f_{1}\left(t_{s}\right)+f_{1}\left(t_{s+1}\right)\right) \\
& +x_{l}\left(-\triangle t \sum_{i=0}^{2 m} a_{i} p_{i, 2}(1)+f_{2}\left(t_{s+1}\right)-f_{1}\left(t_{s+1}-f_{2}\left(t_{s}\right)+f_{1}\left(t_{s}\right)\right)\right. \\
& +u\left(x_{l}, t_{s}\right)\left(\triangle t \sum_{i=0}^{2 m} a_{i} p_{i, 1}\left(x_{l}\right)-\triangle t \sum_{i=0}^{2 m} a_{i} p_{i, 2}(1)+f_{2}\left(t_{s+1}\right)\right. \\
& \left.-f_{1}\left(t_{s+1}\right)+f_{2}\left(t_{s}\right)-f_{1}\left(t_{s}\right)+u^{\prime}\left(x_{l} . t_{s}\right)\right)-2 u\left(x_{l}, t_{s}\right) u^{\prime}\left(x_{l}, t_{s}\right) \\
& =e^{-t_{\mathrm{s}}}\left(\cos x_{l}-\sin x_{l}+\frac{1}{2} e^{-t_{\mathrm{s}}} \sin 2 x_{l}\right) \text {, }
\end{aligned}
$$

which implies that

$$
\begin{aligned}
\triangle & t \sum_{i=0}^{2 m} a_{i} p_{i, 2}\left(x_{l}\right)-x_{l} \sum_{i=0}^{2 m} a_{i} p_{i, 2}(1)-\sum_{i=0}^{2 m} a_{i} h_{i}\left(x_{l}\right)-\Delta t \sum_{i=0}^{2 m} a_{i} h_{i}\left(x_{l}\right) \\
& +\triangle t \sum_{i=0}^{2 m} a_{i} p_{i, 1}\left(x_{l}\right)-\triangle t \sum_{i=0}^{2 m} a_{i} p_{i, 2}(1)+u^{\prime}\left(x_{l}, t_{s}\right) \Delta t \sum_{i=0}^{2 m} a_{i} p_{i, 2}\left(x_{l}\right) \\
& -x_{l} \triangle t \sum_{i=0}^{2 m} a_{i} p_{i, 2}(1)+u\left(x_{l}, t_{s}\right) \triangle t \sum_{i=0}^{2 m} a_{i} p_{i, 1}\left(x_{l}\right)-u\left(x_{l}, t_{s}\right) \Delta t \sum_{i=0}^{2 m} a_{i} p_{i, 2}(1) \\
= & -f_{1}^{\prime}\left(t_{s+1}\right)-x_{l} f_{2}^{\prime}\left(t_{s+1}\right)+x_{l} f_{1}^{\prime}\left(t_{s+1}\right)+u^{\prime \prime}\left(x_{l}, t_{s}\right)-f_{2}\left(t_{s+1}\right) \\
& +f_{1}\left(t_{s+1}\right)-f_{2}\left(t_{s}\right)+f_{1}\left(t_{s}\right)-f_{2}\left(t_{s}\right)-u^{\prime}\left(x_{l}, t_{s}\right)+u^{\prime}\left(x_{l}, t_{s}\right) u\left(x_{l}, t_{s}\right) \\
& +u^{\prime}\left(x_{l}, t_{s}\right) f_{1}\left(t_{s}\right)-u^{\prime}\left(x_{l}, t_{s}\right) f_{1}\left(t_{s+1}\right)+u^{\prime}\left(x_{l}, t_{s}\right) x_{l}\left(f_{2}\left(t_{s+1}\right)-f_{1}\left(t_{s+1}\right)\right) \\
& -u^{\prime}\left(x_{l}, t_{s}\right) x_{l}\left(-f_{2}\left(t_{s}\right)+f_{1}\left(t_{s}\right)\right)+f(x, t) \\
& -u\left(x_{l}, t_{s}\right)\left(f_{2}\left(t_{s+1}\right)-f_{1}\left(t_{s+1}\right)-f_{2}\left(t_{s}\right)-f_{1}\left(t_{s}\right) u^{\prime}\left(x_{l}, t_{s}\right)\right) .
\end{aligned}
$$


Haar wavelets solutions (HWS) are obtained and plots of computed solutions are presented in Figures 4.1a, 4.1b and 4.1c and error norms are also depicted in Table 1.

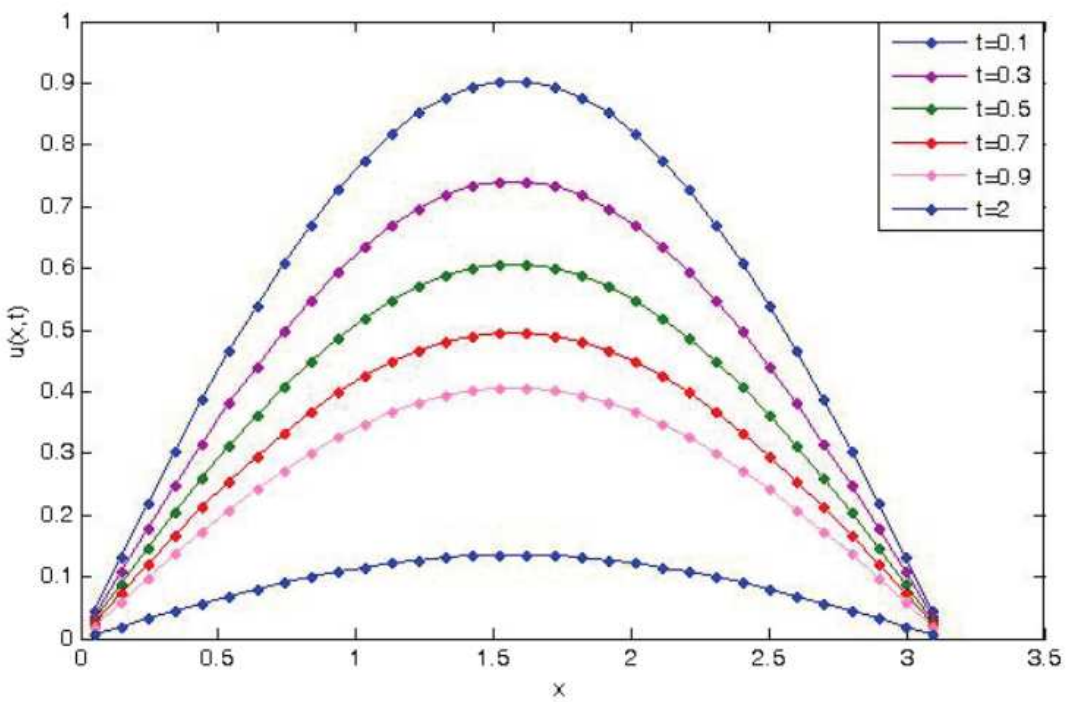

Figure 4.1a: The plot of HWS $u(x, t)$ for $32 \times 32$ grid

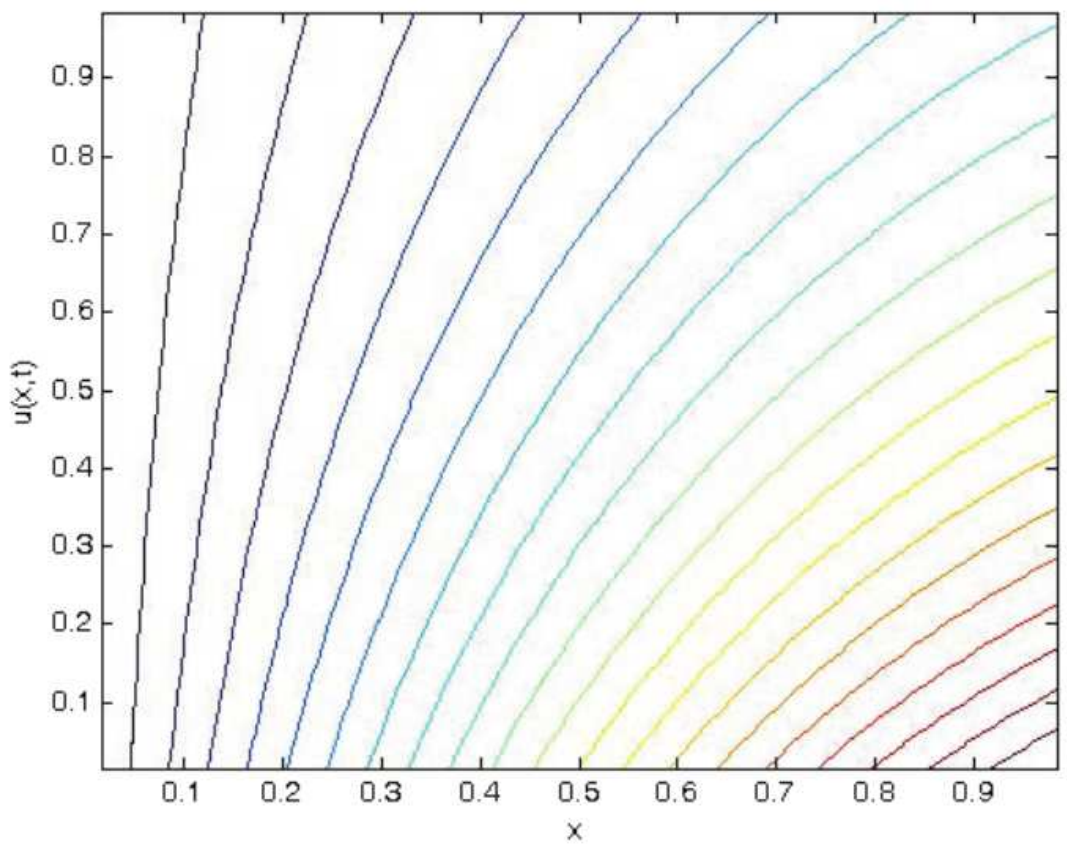

Figure 4.1b: The contour plot of HWS $u(x, t)$ for $32 \times 32$ grid 


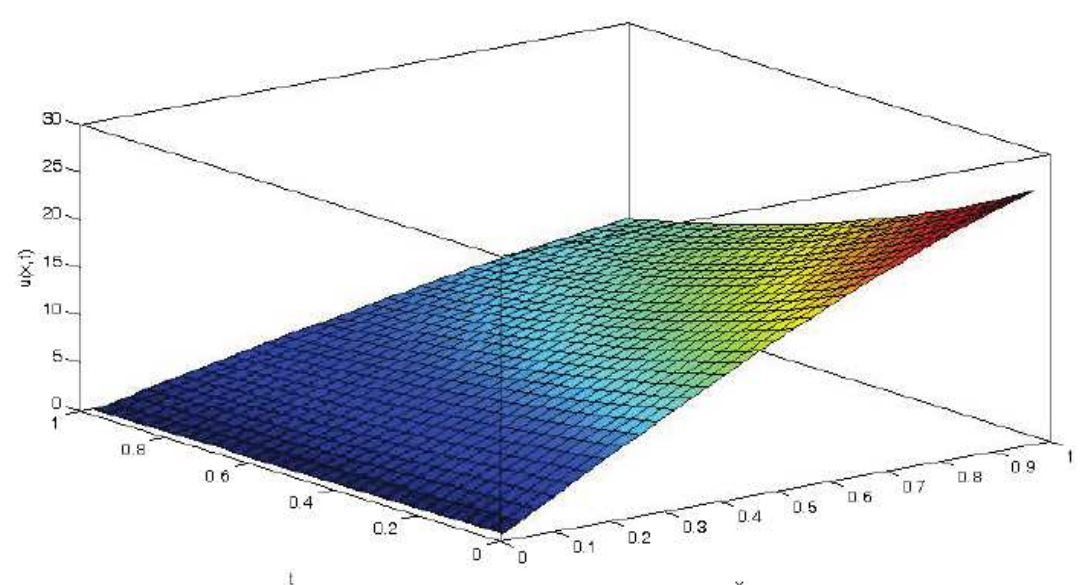

Figure 4.1c: The space time graph of HWS $u(x, t)$ for $32 \times 32$ grid

Example 4.2. (The Damped Generalized Regularized Long Wave Equation)

The nonlinear inhomogeneous damped generalized regularized long wave equation [22] can be written as

$$
u_{t}-\left(\phi(x, t) u_{x t}\right)_{x}-\alpha u_{x x}+\beta u_{x}+u^{p} u_{x}=f(x, t), \quad 0<x \leq 1
$$

with the conditions

$$
u(x, 0)=u_{0}(x), \quad u\left(x_{L}, t\right)=u_{0}\left(x_{R}, t\right)=0, \quad t \in[0, T] .
$$

Consider Eq.(4.2) with initial and boundary conditions as

$$
\begin{aligned}
& u(x, 0)=x\left(x-\frac{1}{2}\right)(x-1), \quad 0 \leq t \leq 1,0 \leq x \leq 1 \\
& u(0, t)=0, \quad u(1, t)=0,
\end{aligned}
$$

and

$$
\begin{aligned}
f(x, t)= & \frac{1}{20} e^{-\frac{1}{10} t}\left(-2 x^{3}+63 x^{2}-181 x+70\right) \\
& \left.+\left(60 x^{5}-150 x^{4}+15 x^{3}-63 x^{2}+19 x-6\right)\right)\left(-\frac{1}{10} t\right) .
\end{aligned}
$$

Solutions are obtained for parameters $\alpha=\beta=p=1$ and $\phi(x, t)=\left(x^{2}+\right.$ 1) $e^{-\frac{1}{10} t}$. The Plots of computed Haar wavelet solutions are presented in Figures $4.2 \mathrm{a}$ and $4.2 \mathrm{~b}$ and error norms are also depicted in Table 1. 


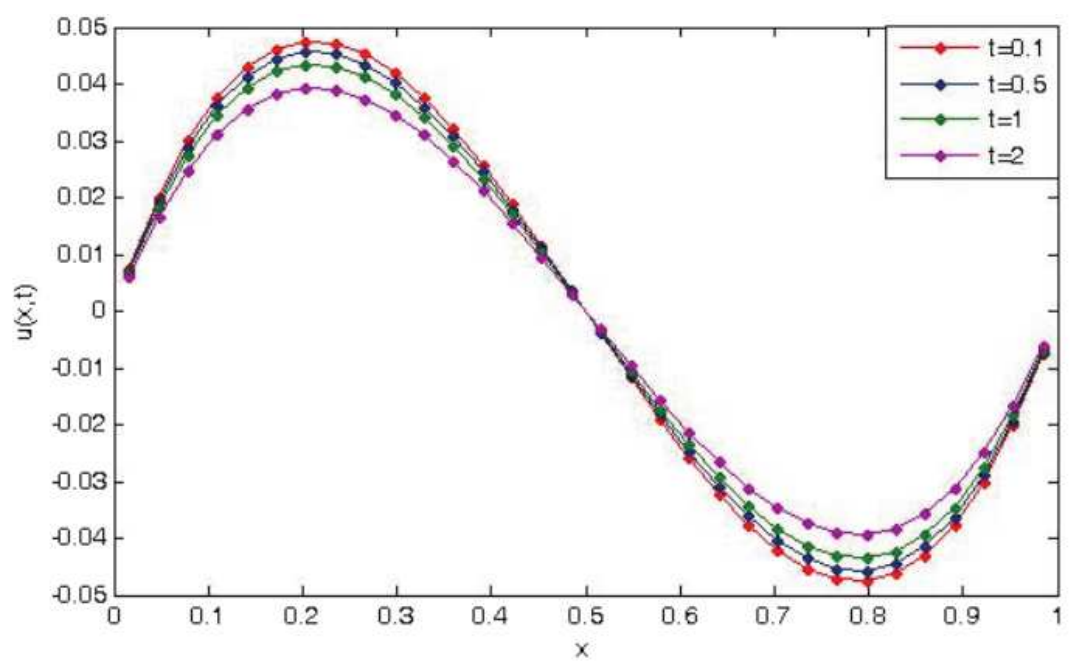

Figure. 4.2a: The plot of HWS $u(x, t)$ for $32 \times 32$ grid

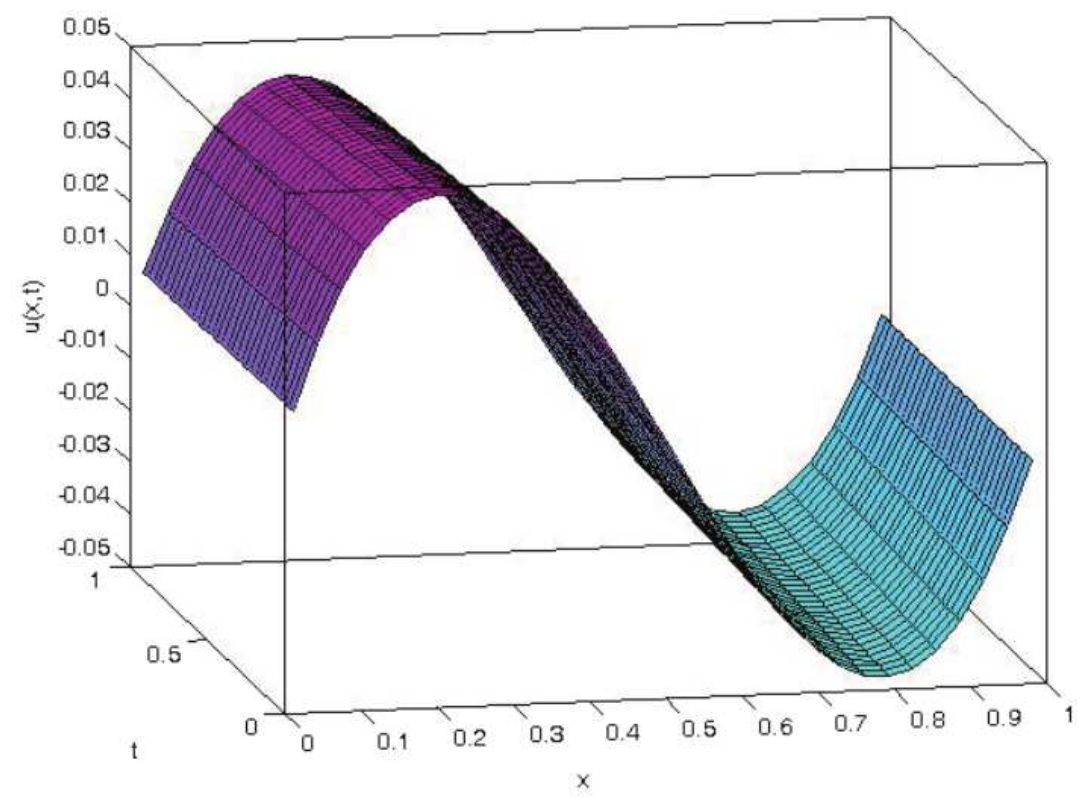

Figure. 4.2b: The space time graph of HWS $u(x, t)$ for $32 \times 32$ grid

Example 4.3. (The Fitzhugh-Nagumo Equation)

The Fitzhugh-Nagumo equation has various applications in the fields of flame propagation, logistic population growth, neurophysiology, branching Brownian motion process, autocatalytic chemical reaction and nuclear reactor theory 
(see [12]). The classical Fitzhugh-Nagumo equation [6] is given by

$$
u_{t}=u_{x x}-u(1-u)(\rho-u), \quad(x, t) \in[A, B] \times[0, T],
$$

where $0 \leq \rho \leq 1$ and $u(x, t)$ is the unknown function depending on the temporal variable $t$ and the spatial variable $x$. This equation combines diffusion and nonlinearity which is controlled by the term $u(1-u)(\rho-u)$. When $\rho=-1$, Eq.(4.3a) reduces to the real Newell-Whitehead equation. The generalized FitzhughNagumo equation with time dependent coefficients and linear dispersion terms given by

$$
u_{t}+\nu(t) u_{x}-\mu(t) u_{x x}-\eta(t) u(1-u)(\rho-u)=0,
$$

where $\nu(t), \mu(t)$ and $\eta(t)$ are arbitrary real-valued functions of $t$.

Consider the nonlinear time-dependent generalized Fitzhugh-Nagumo equation with time-dependent coefficients [4].

$$
u_{t}-\cos t u_{x}-\cos t u_{x x}-2 \cos t u(1-u)(\rho-u)=0
$$

subject to the boundary conditions

$$
\begin{aligned}
& u(A, t)=\frac{\rho}{2}+\frac{\rho}{2} \tanh \left(\frac{\rho}{2}(A-(3-\rho) \sin t)\right), \\
& u(B, t)=\frac{\rho}{2}+\frac{\rho}{2} \tanh \left(\frac{\rho}{2}(B-(3-\rho) \sin t)\right)
\end{aligned}
$$

and the initial condition

$$
u(x, 0)=\frac{\rho}{2}+\frac{\rho}{2} \tanh \frac{\rho x}{2} .
$$

The analytical solution of the equation [4] is given

$$
u(x, t)=\frac{\rho}{2}+\frac{\rho}{2} \tanh \left(\frac{\rho}{2}(x-(3-\rho) \sin t)\right) .
$$




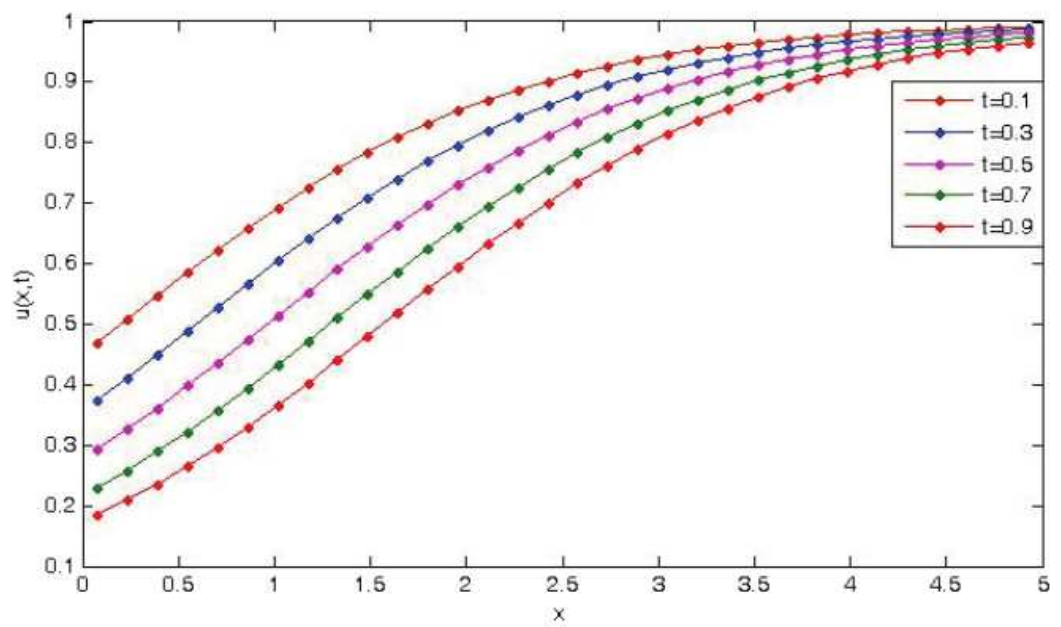

Figure 4.3a: The plot of HWS $u(x, t)$ for $32 \times 32$ grid at different time level

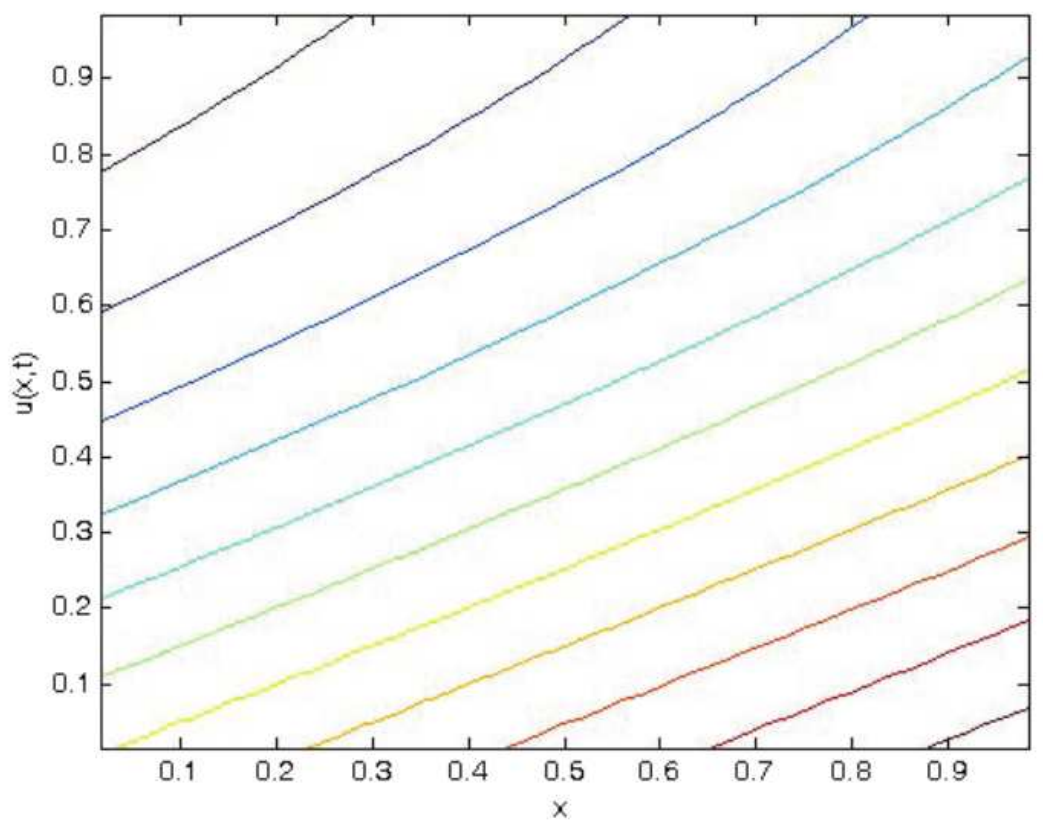

Figure 4.3b: The contour plot of HWS $u(x, t)$ for $32 \times 32$ grid 


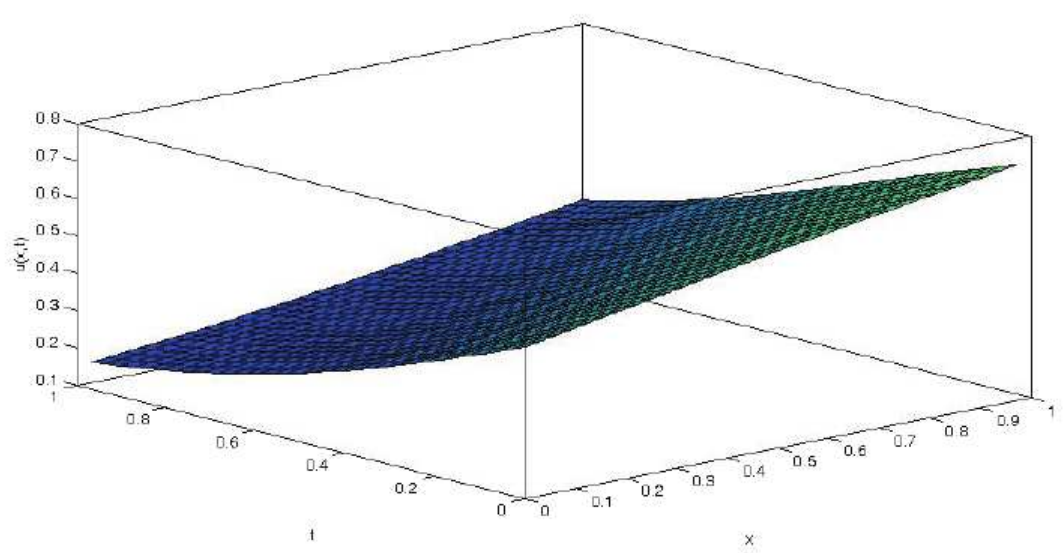

Figure 4.3c: The space time graph of HWS $u(x, t)$ for $32 \times 32$ grid

Solutions are obtained for $A=0, b=1$ and $\rho=1$. The plots of computed Haar wavelet solutions are presented in Figures $4.3 \mathrm{a}, 4.3 \mathrm{~b}$ and $4.3 \mathrm{c}$ and error norms are also depicted in Table 1.

Table 1: Computed error norms for different level of resolution and at different time level

\begin{tabular}{|c|c|c|c|c|c|c|c|}
\hline \multirow{2}{*}{$t$} & \multirow{2}{*}{$\Delta t$} & \multicolumn{2}{|c|}{ Example 4.1} & \multicolumn{2}{c|}{ Example 4.2} & \multicolumn{2}{c|}{ Example 4.3} \\
\cline { 3 - 8 } & & $L_{2}$ & $L_{\infty}$ & $L_{2}$ & $L_{\infty}$ & $L_{2}$ & $L_{\infty}$ \\
\hline 0.1 & 0.0001 & $1.089 \mathrm{E}-3$ & $2.345 \mathrm{E}-4$ & $2.075 \mathrm{E}-3$ & $3.197 \mathrm{E}-4$ & $1.332 \mathrm{E}-3$ & $2.012 \mathrm{E}-4$ \\
0.3 & 0.0001 & $1.167 \mathrm{E}-3$ & $2.402 \mathrm{E}-4$ & $2.175 \mathrm{E}-3$ & $3.274 \mathrm{E}-4$ & $1.472 \mathrm{E}-3$ & $2.114 \mathrm{E}-4$ \\
0.5 & 0.0001 & $1.185 \mathrm{E}-3$ & $2.420 \mathrm{E}-4$ & $2.232 \mathrm{E}-3$ & $3.289 \mathrm{E}-4$ & $1.532 \mathrm{E}-3$ & $2.274 \mathrm{E}-4$ \\
0.7 & 0.0001 & $1.254 \mathrm{E}-3$ & $2.450 \mathrm{E}-4$ & $2.246 \mathrm{E}-3$ & $3.312 \mathrm{E}-4$ & $1.630 \mathrm{E}-3$ & $2.289 \mathrm{E}-4$ \\
0.9 & 0.0001 & $1.267 \mathrm{E}-3$ & $2.513 \mathrm{E}-4$ & $2.289 \mathrm{E}-3$ & $3.410 \mathrm{E}-4$ & $1.694 \mathrm{E}-3$ & $2.312 \mathrm{E}-4$ \\
1.0 & 0.0001 & $1.284 \mathrm{E}-3$ & $2.532 \mathrm{E}-4$ & $2.301 \mathrm{E}-3$ & $3.439 \mathrm{E}-4$ & $1.784 \mathrm{E}-3$ & $2.320 \mathrm{E}-4$ \\
\hline
\end{tabular}

\section{Conclusions}

A time discretization based Haar wavelet numerical schemes is developed to find the numerical solutions of general nonlinear partial differential equations. Quasilinearization in Haar wavelets series overcomes the difficulty to tackle the nonlinearity in nonlinear partial differential equations by converting in a linear system of equations. The scheme is implemented on three test problems of 
well known nonlinear partial differential equations and comparison is also made with available results. Advantage of proposed scheme is that method is easily applicable and reliable to find solutions of general nonlinear partial differential equations in less computation time on selecting collocation points because scheme gives satisfactory numerical results without any iteration. Therefore, it is suggested that quasilinearization process in Haar wavelets based methods can effectively be used to solve the nonlinear partial differential equation.

\section{Acknowledgments}

The authors thankfully acknowledge the valuable discussion and illuminating advice of Prof. R.C. Mittal (Department of Mathematics, Indian Institute of Technology Roorkee, India) which improve the manuscript.

\section{References}

[1] T. Achouri, K. Omrani, Numerical solutions for the damped generalized regularized longwave equation with a variable coefficient by Adomian decomposition method, Commun. Nonlinear Sci. Numer. Simul., 14 (2009), 2025-2033. doi: 10.1016/j.cnsns.2008.07.011

[2] E. Babolian, A. Shahsavaran, Numerical solution of nonlinear Fredholm integrals equations of the second kind using Haar wavelets, J. Comput. Appl. Math., 225 (2009), 87-95. doi: $10.1016 /$ j.cam.2008.07.003

[3] T.B. Benjamin, J.L. Bona, J.J. Mahony, Model equations for long waves in nonlinear dispersive systems, Philos. Trans. Roy. Soc. London Ser. A, 272 (1972), 47-78 doi: 10.1098/rsta.1972.0032

[4] A.H. Bhrawy, A Jacobi-Gauss-Lobatto collocation method for solving generalized Fitzhugh-Nagumo equation with time-dependent coefficients, Appl. Math. Comput., 222 (2013), 255-264. doi: 10.1016/j.amc.2013.07.056

[5] P. Browne, E. Momoniat, F.M. Mahomed, A generalized Fitzhugh-Nagumo equation, Nonlinear Anal., 68 (2008), 1006-1015.

[6] A. Hajipour, S.M. Mahmoudi, Application of exp-function method to Fitzhugh-Nagumo equation, World Appl. Sci. J., 9 (2010), 113-117.

[7] Siraj-ul-Islam, H. Sirajul, A. Arshed, A mesh free method for the numerical solution of the RLW equation, J. Comput. Appl. Math., 223 (2009), 997-1012. doi: 10.1016/j.cam.2008.03.039

[8] H. Kaur, R.C. Mittal, V. Mishra, Haar wavelet approximate solutions for the generalized Lane-Emden equations arising in astrophysics, Comput. Phys. Commun., 184 (2013), 2169-2177. doi: 10.1016/j.cpc.2013.04.013

[9] H. Kaur, R.C. Mittal, V. Mishra, Haar wavelet solutions of nonlinear oscillator equation, Appl. Math. Model., 38 (2014), 4958-4971. doi: 10.1016/j.apm.2014.03.019 
[10] D. Kaya, A Numerical simulation of solitary-wave solutions of the generalized regularized long-wave equation, Appl. Math. Comput., 149 (2004), 833-841. doi: 10.1016/S00963003(03)00189-9

[11] Ü. Lepik, Numerical solution of differential equations using Haar wavelets, Math. Comput. Simulation, 68 (2005), 127-143. doi: 10.1016/j.matcom.2004.10.005

[12] H. Li, Y. Guo, New exact solutions to the Fitzhugh-Nagumo equation, Appl. Math. Comput., 180 (2006), 524-528. doi: 10.1016/j.amc.2005.12.035

[13] V.B. Mandelzweig, F. Tabakin, Quasilinearization approach to nonlinear problems in physics with application to nonlinear ODEs, Comput. Phys. Commun., 141 (2001), 268281. doi: 10.1016/S0010-4655(01)00415-5

[14] L.A. Medeiros, G.P. Menzela, Existence and uniqueness for periodic solutions of the Benjamin-Bona-Mahony equation, SIAM J. Math. Anal., 8 (1977), 792-799. doi: $10.1137 / 0508062$

[15] M. Mei, Large-time behavior of solution for generalized Benjamin-Bona-Mahony-Burgers equations, Nonlinear Anal., 33 (1998) 699-714. doi: 10.1016/S0362-546X(97)00674-3

[16] R.C. Mittal, H. Kaur, V. Mishra, Haar wavelet based numerical investigation of coupled viscous Burgers equation, Int. J. Comput. Math., 92 (2015), 1643-1659. doi: 10.1080/00207160.2014.957688

[17] M. Mohammadi, R. Mokhtari, Solving the generalized regularized long wave equation on the basis of a reproducing kernel space, J. Comput. Appl. Math., 235 (2011), 4003-4014. doi: $10.1016 /$ j.cam.2011.02.012

[18] J.S. Nagumo, S. Arimoto and S. Yoshizawa, An active pulse transmission line simulating nerve axon, Proc. IRE 50 (1962), 2061-2070.

[19] K. Omrani, M. Ayadi, Finite difference discretization of the Benjamin-Bona-MahonyBurgers equation, Numer. Methods Partial Differential Equations, 24 (2008), 239-248. doi: $10.1002 /$ num.20256

[20] W. Sweldens, The Construction and application of wavelets in numerical analysis, Ph.D Thesis, 1995.

[21] H. Triki, A.M. Wazwaz, On soliton solutions for the Fitzhugh-Nagumo equation with time-dependent coefficients, Appl. Math. Model., 37 (2013), 3821-3828. doi: 10.1016/j.apm.2012.07.031

[22] S.A. Yousefi, Z. Barikbin, M. Behroozifar, Bernstein Ritz-Galerkin method for Solving the damped generalized regularized long-wave (DGRLW) equation, Int. J. Nonlinear Sci., 9 (2010), 151-158. 